\title{
Case Report \\ Obsessive-Compulsive Disorder with Suicide Obsessions in a First Responder without Previous Diagnosis of OCD or History of Suicide Attempts
}

\author{
Vivekananda Rachamallu, ${ }^{1}$ Michael M. Song, ${ }^{2}$ Haiying Liu, ${ }^{3}$ \\ Charles L. Giles, ${ }^{1}$ and Terry McMahon ${ }^{1}$ \\ ${ }^{1}$ Texas Tech University Health Sciences Center School of Medicine, Department of Psychiatry, Lubbock, TX, USA \\ ${ }^{2}$ Texas Tech University Health Sciences Center School of Medicine and Graduate School of Biomedical Sciences, \\ MD/PhD Program, Lubbock, TX, USA \\ ${ }^{3}$ Texas Tech University Health Sciences Center School of Medicine, Lubbock, TX, USA
}

Correspondence should be addressed to Vivekananda Rachamallu; vivekananda.rachamallu@ttuhsc.edu

Received 24 April 2017; Revised 23 July 2017; Accepted 24 August 2017; Published 2 October 2017

Academic Editor: Erik Jönsson

Copyright (C) 2017 Vivekananda Rachamallu et al. This is an open access article distributed under the Creative Commons Attribution License, which permits unrestricted use, distribution, and reproduction in any medium, provided the original work is properly cited.

\begin{abstract}
Obsessive-compulsive disorder (OCD) is a distressing and often debilitating disorder characterized by obsessions, compulsions, or both that are time-consuming and cause impairment in social, occupational, or other areas of functioning. There are many published studies reporting higher risk of suicidality in OCD patients, as well as studies describing increased risk of suicidality in OCD patients with other comorbid psychiatric conditions such as major depressive disorder (MDD) and posttraumatic stress disorder (PTSD). Existing case reports on OCD with suicide as the obsessive component describe patients with long standing diagnosis of OCD with suicidal ideations or previous suicide attempts. This report describes the case of a 28 -year-old male, who works as a first responder, who presented with new onset symptoms characteristic of MDD and PTSD, with no past history of OCD or suicidality who developed OCD with suicidal obsessions. Differentiating between suicidal ideation in the context of other psychiatric illnesses and suicidal obsessions in OCD is critical to ensuring accurate diagnosis and timely provision of most appropriate treatment. The combination of exposure and response prevention therapy and pharmacotherapy with sertraline and olanzapine was effective in helping the patient manage the anxiety and distress stemming from the patient's OCD with suicidal obsession.
\end{abstract}

\section{Introduction}

The cardinal features of obsessive-compulsive disorder (OCD) include obsessions, compulsions, or both that are time-consuming and cause clinically significant distress or impairment in social, occupational, or other areas of functioning [1]. Obsessions are repetitive, intrusive, and unwanted thoughts or images (often of sexual, religious, aggressive, or death-related nature) that cause significant anxiety and distress [2]. Compulsions or rituals are repetitive behaviors or mental acts that are performed by those with OCD, in an attempt to decrease their anxiety or distress [1]. There are many published studies reporting higher risk of suicidality in OCD patients, as well as studies describing increased risk of suicidality in OCD patients with other comorbid psychiatric conditions such as major depressive disorder (MDD) and posttraumatic stress disorder (PTSD) [3-11]. Furthermore, violent obsessions have been reported to be positively correlated with suicidality in OCD $[9,12]$. However, OCD with ego-dystonic suicidal obsessions have rarely been reported [13-15]. Al-Zaben described a patient with long history of both MDD and OCD who developed suicidal obsessions [13] while Wetzler et al. described the development of OCD with suicidal obsessions in a patient with MDD, following a suicide attempt [14]. Furthermore, Wetterneck et al. described OCD with suicidal obsession in a patient with treatment-resistant OCD [15] (see Table 1). This report describes the case of a 28year-old male, who works as a first responder, who presented 
TABLE 1: Previously published case reports on OCD with suicidal obsession.

\begin{tabular}{lcccc}
\hline Report & Age & Sex & History & Comments \\
\hline Al-Zaben [13] & 54 & Female & $\begin{array}{c}\text { Long history of MDD, } \\
\text { long history of OCD }\end{array}$ & $\begin{array}{c}\text { OCD symptoms were improved with paroxetine and } \\
\text { cognitive restructuring. }\end{array}$ \\
\hline Wetzler et al. [14] & 29 & Female & $\begin{array}{c}\text { Long history of MDD, } \\
\text { suicide attempt with } \\
\text { posttraumatic obsession }\end{array}$ & $\begin{array}{c}\text { Depression was improved with ECT and citalopram. } \\
\text { OCD improved with gradual exposure therapy. }\end{array}$ \\
\hline Wetterneck et al. [15] & 40s & Male & $\begin{array}{c}\text { MDD, chronic treatment } \\
\text { resistant OCD }\end{array}$ & $\begin{array}{c}\text { Improved after treatment with Foa et al.s protocol on } \\
\text { exposure and ritual/response prevention (ERP), an } \\
\text { evidence-based treatment for OCD. }\end{array}$ \\
$\begin{array}{l}\text { Aukst-Margetić et al. } \\
{[16]}\end{array}$ & 54 & Male & Bipolar disorder & $\begin{array}{c}\text { Developed ego-dystonic suicidal obsession with clozapine in } \\
\text { a dose dependent manner. Improved with switch to a } \\
\text { different atypical antipsychotic. }\end{array}$ \\
\hline
\end{tabular}

with symptoms characteristic of MDD and PTSD, with no past history of OCD or suicidality, with newly developed OCD with suicidal obsessions.

\section{Case Description}

A 28-year-old Caucasian male was brought to the emergency department (ED) by his wife and parents due to sudden onset, intense suicidal ideations. He had no significant past medical or psychiatric history except for a 6-month history of depressed mood and anxiety in the context of several stressors in his family and at work. The family reported that the patient developed blunted affect and significantly reduced vocalization over those 6 months. He did not have any prior history of suicide attempts or psychiatric diagnoses or hospitalizations. He reported drinking 3 beers up to 4 times a week but he denied any other history of substance abuse or any family history of psychiatric problems. Following the evaluation at the ED, the patient was admitted to the inpatient psychiatric care unit with suicide precautions. During the initial inpatient evaluation, the patient reported feeling depressed and anxious, as well as having episodic nightmares once every few months due to having witnessed many scenes of death as a firefighter, including a suicide by hanging and violent car wrecks. He reported that, for the previous 3 months, he had been having thoughts about killing himself which was worsened with seeing firearms, razor blades, or pocket knife. He reported that the thoughts of suicide started after witnessing the carnage of a car wreck 3 months before. Earlier in the day, prior to admission, he had held an unloaded gun to his head, which was witnessed by his family and was the ultimate reason the family brought him to the ED. He also reported not being able to relax, feeling on edge, and feeling like he should always be doing something. All potential medical or substance related causes of the patient's psychiatric condition were ruled out. The initial list of differential diagnoses included major depressive disorder (MDD), bipolar disorder, and cyclothymia (due to the reported symptoms of depression and impulsivity). The patient's progress through the initial hospital admission is outlined in Table 2. The patient was ultimately discharged with olanzapine, divalproex extended release, prazosin, clonazepam, and appointments for a therapist and an outpatient psychiatrist.
Two days after being discharged from the inpatient psychiatric care unit, the patient was readmitted with continuing suicidal thoughts which he was unable to control. The patient reported that, during the previous admission, he had told the clinical staff that his symptoms were improving so that could be discharged from the hospital. During the ensuing interview, the patient described in a greater detail the nature of his suicidal thoughts. He reported that he was having sudden onset, intense, recurrent (5-6 times a day), short lasting (a few minutes each), anxiety provoking, unwanted, and distressing thoughts of committing suicide for the past 3 months, since witnessing a car wreck. He reported that he did not believe that his family would be better off without him and that thinking about what his death would do to his wife also caused him significant anxiety and distress. He reported that he rapidly became overwhelmed and panicked with each intense thought of suicide. The patient's social and occupational life was significantly affected due to the obsessive thoughts, becoming more isolated from his family members and skipping work due to fear of triggering suicidal obsessive thoughts. The patient denied any compulsive rituals or repetitive behaviors to reduce the anxiety in relation to obsessive suicidal thoughts. However, he did report trying to block those thoughts by distracting himself, attempting to redirect his thoughts, or seeking reassurance from his family. He expressed a sense of hopelessness and helplessness regarding the intrusive suicidal thoughts. He also reported low self-esteem and negative self-image due to his inability to control those distressing thoughts. He said, as the thoughts continued to return, he did think about acting on those thoughts to make them stop. At the same time, he noted that harming himself was not something that he wanted to do and that he wanted to get help so that he would not actually do so. Once again, all potential medical or substance related causes of the patient's psychiatric condition were ruled out and according to the Diagnostic Statistical Manual of Mental Disorders, 5th ed. (DSM-V), the patient was diagnosed with OCD with obsessive suicidal thoughts without compulsions.

The patient was started on a pharmacotherapy regimen including sertraline $25 \mathrm{mg}$ oral tablet once daily for OCD, PTSD, and MDD, olanzapine $10 \mathrm{mg}$ oral tablet at bedtime for impulse control, and clonazepam $0.5 \mathrm{mg}$ oral tablet three times a day for anxiety. In the ensuing days, the patient 


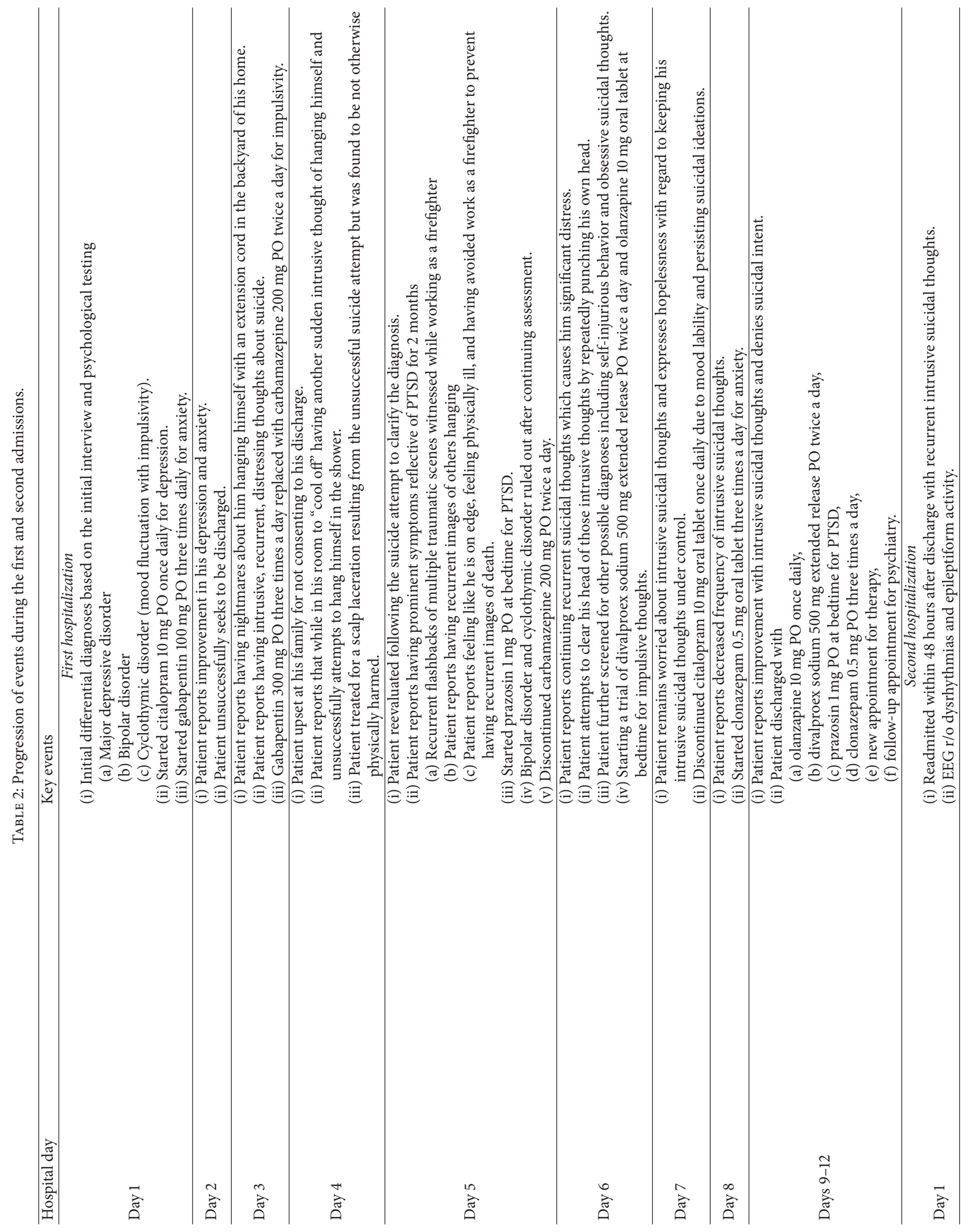




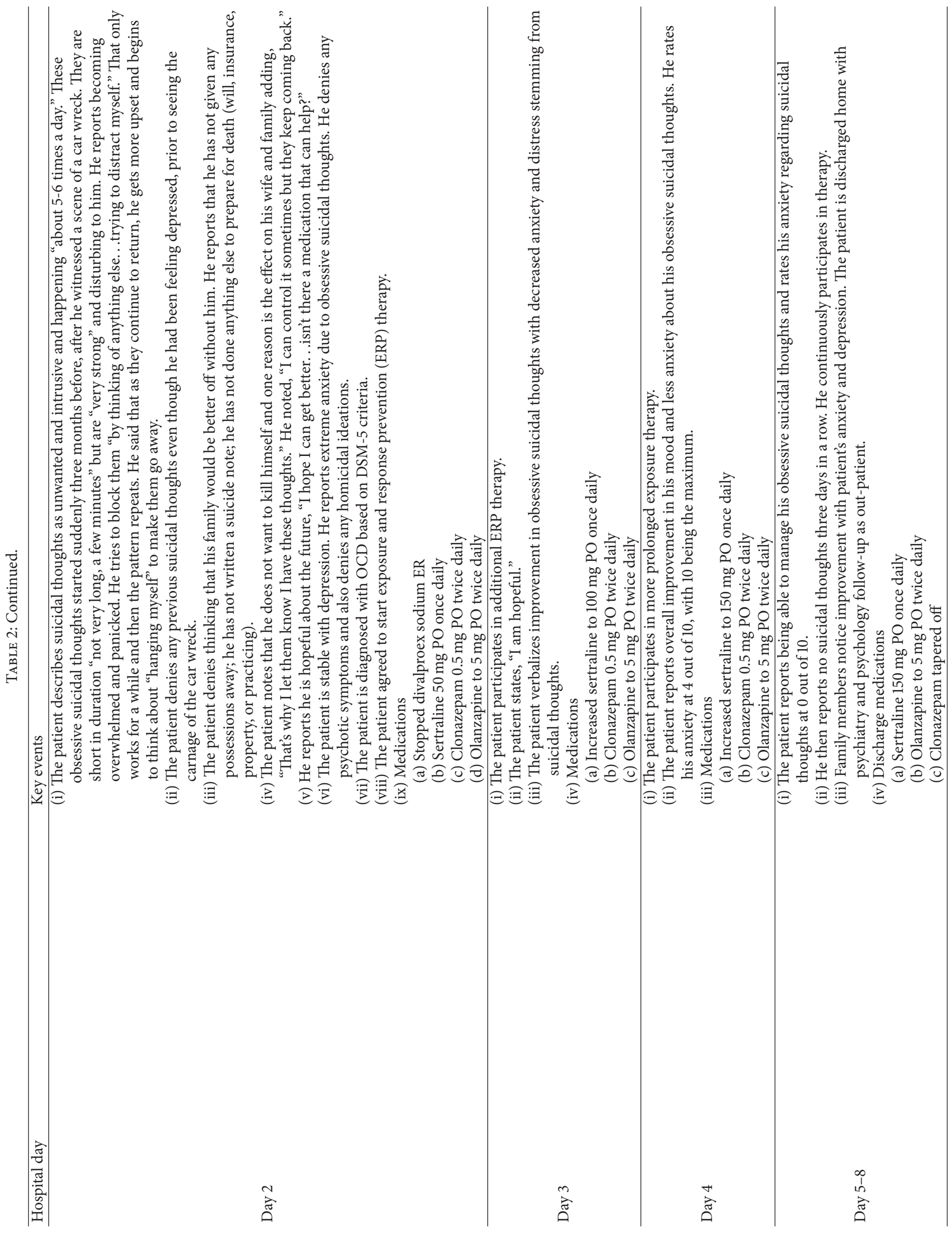


was treated with exposure and response prevention (ERP) therapy, an effective cognitive-behavioral therapy for OCD. During the ERP therapy sessions, the patient was instructed to focus on a suicidal thought, report the level of anxiety to the therapist, and avoid calling for an ambulance or calling his wife for reassurance. The patient's anxiety levels were high during the initial sessions of ERP. Motivational interviewing techniques were used to provide encouragement and support in helping the patient continue the therapy. Once the patient's anxiety was decreased and the thought became nondistressing, the patient was encouraged to focus on a different suicidal thought and continue pondering on those thoughts within the ERP therapy regimen, until the thought was no longer distressing. The patient received multiple ERP sessions during the hospitalization, each with different types of suicidal thoughts. Later ERP sessions included cues to more disturbing suicidal thoughts. By the end of the hospitalization, the patient reported a change in his relationship with the obsessive suicidal thoughts and learned that he did not have to engage in suppression or avoidant strategies when those thoughts occurred.

Over the next several days, his sertraline dose was also gradually increased to $150 \mathrm{mg}$ once daily. The patient continued on sertraline, olanzapine, clonazepam, and individual CBT. He reported a decrease in the occurrence of his obsessive suicidal thought, decreased distress when it did appear, and no further need for avoidant behaviors related to the thought. The patient gradually became more verbal, his affect returned to full range, and he actively began participating in group therapy sessions. The clonazepam was tapered off and the patient was discharged on hospital day 8 , with sertraline and olanzapine. He was scheduled for follow-up with an individual therapist to help him continue to develop skills to better manage his anxiety and obsessive suicidal thoughts.

\section{Discussion}

The Diagnostics and Statistics Manual of Mental Disorders, 5th ed. (DSM-5), defines suicidal ideation as "thoughts about self-harm, with deliberate consideration or planning of possible techniques of causing one's own death.” Rumination of suicidal ideation in mood disorders is generally moodcongruent and not necessarily experienced as intrusive, distressing, or linked to compulsions [1]. Suicidal obsession on the other hand is characterized by recurrent, persistent, intrusive, and unwanted thoughts about suicide that cause marked anxiety or distress $[1,13,14]$. Factors suggesting OCD related thoughts include ego-dystonicity, absence of past behavior consistent with the thought, presence of avoidance behavior (i.e., avoidance of any tools of suicide), frequent thoughts, high degree of distress, and fair-good insight with strong motivation to seek help [17].

Patients with OCD have been reported to be at a greater risk of suicide than the general population [3-11]. Furthermore, suicidal risk in patients with OCD has been reported to be worsened by comorbid psychiatric conditions or OCD with aggressive or violent obsessions [9-12]. However, it is important to recognize that suicidal thoughts in patients with OCD can be an obsession of self-harm that is distinct from factual suicidal ideations. OCD with ego-dystonic suicidal obsessions have rarely been reported in the literature (see Table 1). Each previously reported case of OCD with suicidal obsessions has been in patients with history of long standing OCD, treatment refractory OCD, or history of suicide attempt (Table 1) [13-15]. The case report by Al-Zaben [13] describes a patient with suicidal obsession in long standing OCD with comorbid depression. A case report by Wetzler et al. [14] described a patient with long standing MDD who developed obsessive thoughts of harming herself following a failed suicide attempt (posttraumatic obsession). In addition, a case report by Watterneck et al. [15] described suicidal obsession in a patient with chronic treatment-resistant OCD with comorbid depression who was successfully treated with methodology described by Foa et al. [18], an evidence-based treatment for OCD. There is also a case report describing egodystonic suicidal obsessions occurring as a dose-dependent side-effect of clozapine [16].

The patient described in this case report is unique in that, unlike previously reported cases of OCD with suicidal obsessions, he did not have previous history of any chronic neuropsychiatric diagnoses or any cognitive-behavioral issues. In addition, our patient did not demonstrate any compulsive rituals to reduce his anxiety caused by his obsessive suicidal thoughts. Approximately 25\% of OCD patients report distressing obsessions without overt compulsive rituals [19]. The most common comorbid psychiatric diagnoses associated with OCD are depression, anxiety, bipolar affective disorder, and attention deficit hyperactivity disorder [20, 21]. Our patient was assessed for comorbidities and other obsessivecompulsive related disorders (OCRD). He was ultimately diagnosed with new onset symptoms of OCD with suicidal obsessions, with comorbid depression, anxiety, and PTSD, all of which were of new onset. Furthermore, the patient did not have any history of violent acts towards self or others. His exposure to suicide and traumatic death was strictly as a witness while on duty as a first responder. Nevertheless, he developed symptoms of depression and PTSD, as well as obsessive suicidal thoughts.

Although our patient exhibited symptoms consistent with MDD and PTSD, his suicidal thoughts were OCD related, with frequent and intrusive, ego-dystonic, and highly distressing suicidal thoughts that were also associated with avoidance behavior and desperate desire for relief from the illogical, obsessive thoughts. Our patient reported that he actually did not want to harm himself and therefore, the recurrent thoughts of suicide were highly anxiety provoking and distressing. He reported that he did not believe that his family would be better off without him and that thinking about what his death would do to his wife also caused him significant anxiety and distress. He also reported that he rapidly became overwhelmed and panicked with each intense thought of suicide. His suicidal ideations were therefore different in nature than "mood-congruent" suicidal ideations observed in uncontrolled severe MDD patients. In addition, he described that his attempts at suicide (holding an unloaded gun to his head prior to admission and unsuccessfully attempting to hang himself with a towel in the shower while at the hospital) were the result of him being unable to redirect 
his thoughts away from the obsessive suicidal thoughts, ultimately resulting in him acting out those thoughts. Although his suicidal behavior may stem from poor impulse control, they were not compulsions or behavior stemming from poor insight in believing that his family would be better off without him.

Such an unusual initial presentation of OCD, combined with concurrent symptoms of PTSD and mood disorders (which was the initial chief complaint during the first presentation at the emergency department), posed a significant challenge in the accurate diagnosis of the patient's condition, with potentially catastrophic consequences to the patient's wellbeing. A thorough exploration of the origin of the patient's suicidal thoughts is essential to the diagnosis of $\mathrm{OCD}$ with suicidal obsessions.

Evidence-based psychotherapy protocols for separate diagnoses have been utilized successfully as a standard for the treatment of a variety of disorders. To address the challenge that arises with treatment of multiple disorders, a Unified Protocol for Emotional Disorders (UP) was developed, largely due to the growing body of research supporting commonalities in many of these disorders [22]. The CBT techniques used across depression and anxiety converge into the core principle of detecting the unique unwanted thoughts, physical sensations, and emotions, as well as the avoidant strategies and negatively reinforce the failed strategy used by the patient. Ultimately, purposeful exposure to the avoided material becomes a core treatment component for therapy with the anxiety and depressive disorders.

The key issue in our case was determining whether the suicidal thoughts and actions were themselves amenable to exposure and avoidance prevention strategies. The unwanted nature of the thought, the connection of the thought to sympathetic arousal, and the reinforcing compulsive behaviors led to the decision to provide a trial of exposure therapy. The treatment utilized, exposure and response therapy (ERP, a form of CBT), combined with pharmacotherapy with a serotonin reuptake inhibitor (SSRI) plus antipsychotics, as described by Seibell and Hollander [2] for the treatment of OCD, was effective in helping the patient manage the anxiety and distress stemming from his OCD with suicidal obsession.

\section{Conclusion}

In summary, OCD with suicidal obsessions is a rarely reported presentation of OCD. Differentiating between suicidal ideation in the context of psychiatric illnesses and suicidal obsessions in OCD is critical to ensuring accurate diagnosis of the patient's condition, as well as timely provision of most appropriate multidisciplinary treatment. As described in this case report, new onset OCD with suicide obsession should not be neglected in the differential in those with newly developing symptoms of OCD and other comorbid psychiatric conditions, especially in those with history of exposure to scenes of suicide and death in personal or professional setting. Patients presenting with suicidal ideation and diagnosed with OCD must undergo a meticulous risk assessment to differentiate suicidal obsession from suicidal ideations, especially when there are other comorbid psychiatric conditions.
Furthermore, it is important to assess suicidal ideations in OCD patients with obsessive thoughts of violence, as they are at a higher risk of self-harm [12]. The ability to accurately separate the obsession is critical to provide effective treatment. Therefore, a thorough exploration of the origin of the patient's suicidal thoughts is essential to the accurate diagnosis and timely provision of optimal multidisciplinary treatment of OCD with suicidal obsessions. In the case of our patient, ERP combined with sertraline and olanzapine, which matches the recommended standard of care for OCD with CBT, SSRI, and antipsychotic $[2,23,24]$, was effective in the management of his symptoms of OCD with suicidal obsession.

\section{Conflicts of Interest}

The authors report no conflicts of interest.

\section{Authors' Contributions}

Vivekananda Rachamallu, Michael M. Song, Haiying Liu, Charles L. Giles, and Terry McMahon organized and prepared this manuscript.

\section{References}

[1] American Psychiatric Association, Diagnostic and Statistical Manual of Mental Disorders, DSM-5, American Psychiatric Association, Arlington, tex, USA, 5th edition, 2013.

[2] P. J. Seibell and E. Hollander, "Management of obsessivecompulsive disorder," F1000Prime Reports, vol. 6, article no. 68, 2014.

[3] I. Angelakis, P. Gooding, N. Tarrier, and M. Panagioti, "Suicidality in obsessive compulsive disorder (OCD): A systematic review and meta-analysis," Clinical Psychology Review, vol. 39, pp. 1-15, 2015.

[4] L. Fernández de la Cruz, M. Rydell, B. Runeson et al., "Suicide in obsessive-compulsive disorder: a population-based study of 36788 Swedish patients," Molecular Psychiatry, 2016.

[5] P. Velloso, C. Piccinato, Y. Ferrão et al., "The suicidality continuum in a large sample of obsessive-compulsive disorder (OCD) patients," European Psychiatry, vol. 38, pp. 1-7, 2016.

[6] A. R. Torres, A. T. A. Ramos-Cerqueira, Y. A. Ferrão, L. F. Fontenelle, M. C. Do Rosário, and E. C. Miguel, "Suicidality in obsessive-compulsive disorder: Prevalence and relation to symptom dimensions and comorbid conditions," Journal of Clinical Psychiatry, vol. 72, no. 1, pp. 17-26, 2011.

[7] P. Alonso, C. Segalàs, E. Real et al., "Suicide in patients treated for obsessive-compulsive disorder: A prospective follow-up study," Journal of Affective Disorders, vol. 124, no. 3, pp. 300-308, 2010.

[8] P. Kamath, Y. C. J. Reddy, and T. Kandavel, "Suicidal behavior in obsessive-compulsive disorder," Journal of Clinical Psychiatry, vol. 68, no. 11, pp. 1741-1750, 2007.

[9] G. Gupta, A. Avasthi, S. Grover, and S. M. Singh, "Factors associated with suicidal ideations and suicidal attempts in patients with obsessive compulsive disorder," Asian Journal of Psychiatry, vol. 12, no. 1, pp. 140-146, 2014.

[10] J. E. DeVylder, A. J. Oh, S. Ben-David, N. Azimov, J. M. HarkavyFriedman, and C. M. Corcoran, "Obsessive compulsive symptoms in individuals at clinical risk for psychosis: Association 
with depressive symptoms and suicidal ideation," Schizophrenia Research, vol. 140, no. 1-3, pp. 110-113, 2012.

[11] L. F. Fontenelle, L. Cocchi, B. J. Harrison et al., "Towards a posttraumatic subtype of obsessive-compulsive disorder," Journal of Anxiety Disorders, vol. 26, no. 2, pp. 377-383, 2012.

[12] T. H. W. Ching, M. Williams, and J. Siev, "Violent obsessions are associated with suicidality in an OCD analog sample of college students," Cognitive Behaviour Therapy, vol. 46, no. 2, pp. 129140, 2017.

[13] F. Al-Zaben, "Suicidal Obsessions in a Patient with Obsessive Compulsive Disorder: A Case Report," Journal of King Abdulaziz University-Medical Sciences, vol. 19, no. 4, pp. 121-127, 2012.

[14] A. J. Wetzler, R. Elias, L. Fostick, and J. Zohar, "Suicidal ideation versus suicidal obsession: A case report," CNS Spectrums, vol. 12, no. 7, pp. 553-556, 2007.

[15] C. T. Wetterneck, M. T. Williams, G. Tellawi, and S. L. Bruce, "Treatment of suicide obsessions in obsessive-compulsive disorder with comorbid major depressive disorder," Clinical Handbook of Obsessive-Compulsive and Related Disorders: A CaseBased Approach to Treating Pediatric and Adult Populations, pp. 431-445, 2015.

[16] B. Aukst-Margetić, B. Margetić, and V. Boričević Maršanić, "Suicidal obsessions as dose dependent side-effect of clozapine," Psychopharmacol Bull, vol. 44, no. 1, pp. 65-69, 2011.

[17] D. Veale, M. Freeston, G. Krebs, I. Heyman, and P. Salkovskis, "Risk assessment and management in obsessive-compulsive disorder," Advances in Psychiatric Treatment, vol. 15, no. 5, pp. 332-343, 2009.

[18] E. B. Foa, E. Yadin, and T. K. Lichner, Exposure and Response (Ritual) Prevention for Obsessive Compulsive Disorder, Oxford University Press, 2012.

[19] D. McKay, J. S. Abramowitz, J. E. Calamari et al., "A critical evaluation of obsessive-compulsive disorder subtypes: Symptoms versus mechanisms," Clinical Psychology Review, vol. 24, no. 3, pp. 283-313, 2004.

[20] D. L. Murphy, K. R. Timpano, M. G. Wheaton, B. D. Greenberg, and E. C. Miguel, "Obsessive-compulsive disorder and its related disorders: A reappraisal of obsessive-compulsive spectrum concepts," Dialogues in Clinical Neuroscience, vol. 12, no. 2, pp. 131-148, 2010.

[21] G. Perugi, C. Toni, F. Frare, M. C. Travierso, E. Hantouche, and H. S. Akiskal, "Obsessive-compulsive-bipolar comorbidity: A systematic exploration of clinical features and treatment outcome," Journal of Clinical Psychiatry, vol. 63, no. 12, pp. 11291134, 2002.

[22] C. F. Sharpley, "Clinical handbook of psychological disorders: A step by step treatment manual,Barlow David H. (Ed.),New York:The Guilford Press,1985,586 pp.; \$A69.80," Behaviour Change, vol. 5, no. 03, pp. 139-140, 1988.

[23] S. Dimidjian, S. D. Hollon, K. S. Dobson et al., "Randomized trial of behavioral activation, cognitive therapy, and antidepressant medication in the acute treatment of adults with major depression," Journal of Consulting and Clinical Psychology, vol. 74, no. 4, pp. 658-670, 2006.

[24] D. Veale, "Behavioural activation for depression," Advances in Psychiatric Treatment, vol. 14, no. 1, pp. 29-36, 2008. 


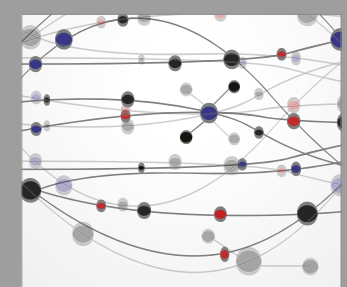

The Scientific World Journal
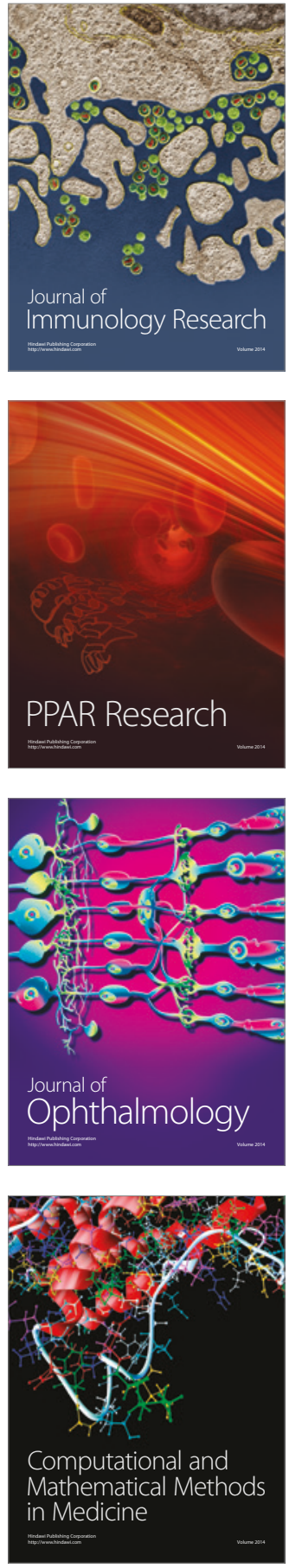

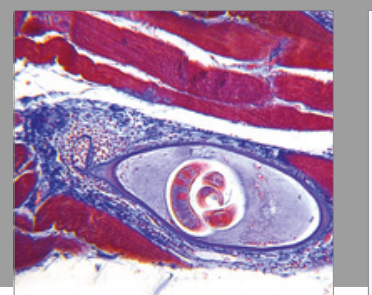

Gastroenterology Research and Practice
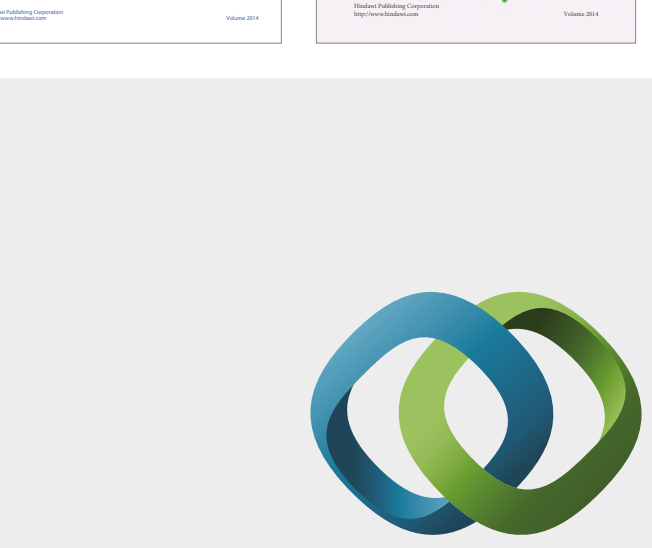

\section{Hindawi}

Submit your manuscripts at

https://www.hindawi.com
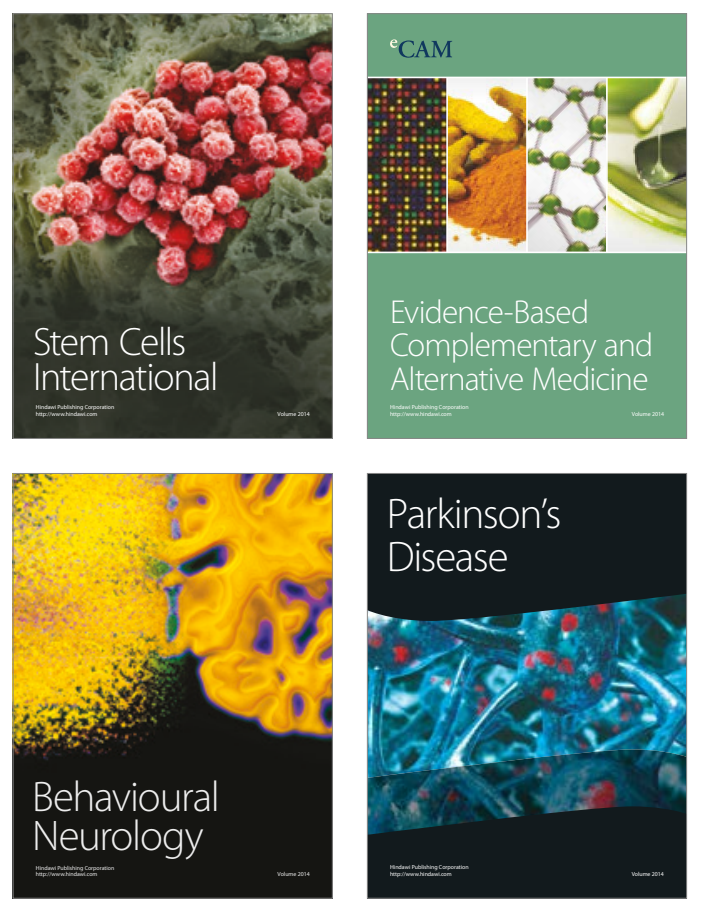
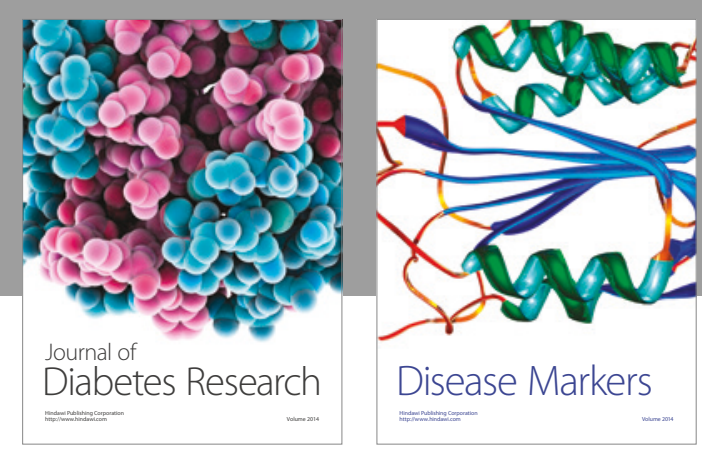

Disease Markers
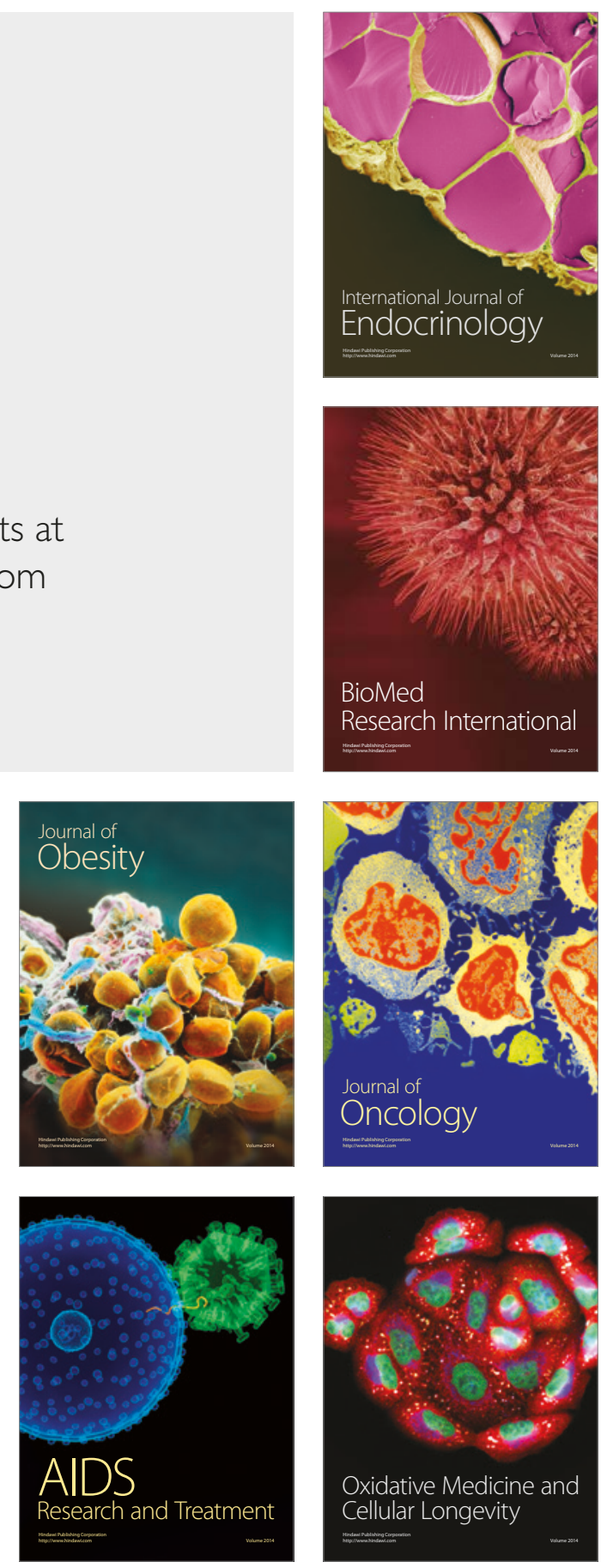